\title{
Population interaction structure and the coexistence of bacterial strains playing 'rock-paper-scissors'
}

\author{
Robert A. Laird \\ R. A. Laird (robert.laird@uleth.ca),Dept of Biological Sciences, Univ. of Lethbridge, Lethbridge, AB, T1K 3M4, Canada.
}

\begin{abstract}
The simplest example of non-transitive competition is the game rock-paper-scissors (RPS), which exhibits characteristic cyclic strategy replacement: paper beats rock, which in turn beats scissors, which in turn beats paper. In addition to its familiar use in understanding human decision-making, rock-paper-scissors is also played in many biological systems. Among other reasons, this is important because it potentially provides a mechanism whereby species- or strain coexistence can occur in the face of intense competition. Kerr et al. (2002, Nature 418: 171-174) use complementary experiments and simulations to show that RPS-playing toxic, resistant, and susceptible E. coli bacteria can coexist when interactions between the strains are spatially explicit. This raises the question of whether limited interactions associated with space are sufficient to allow strain coexistence, or whether space per se is crucial. I approach this question by extending the Kerr et al. model to include different (aspatial) population network structures with the same degree distributions as corresponding spatial lattice models. I show that the coexistence that occurs for some parameter combinations when simulated bacterial strains compete on lattices is absent when they compete on random regular graphs. Further, considering small-world networks of intermediate 'quenched randomness' between lattices and random regular graphs, I show that only small deviations from pure spatial interactions are sufficient to prevent strain coexistence. These results emphasize the explicit role of space, rather than merely limited interactions, as being decisive in allowing the coexistence of toxic, resistant, and susceptible strains in this model system.
\end{abstract}

One of the main goals of community ecology is to determine how species or strains can coexist in the face of intense competition (Wilson 1990, Tokeshi 1999, Chesson 2000). Most proposed mechanisms of coexistence involve the mitigation of competition, for example by niche differentiation or disturbance. In contrast, here I discuss a situation where competition - more specifically, non-transitive competition - is not an obstacle that must be overcome, but rather is a promoter of coexistence in its own right.

The simplest example of non-transitive competition is the game of rock-paper-scissors (RPS), in which rock is covered by paper, paper is cut by scissors, and scissors is crushed by rock $(\mathrm{R} \rightarrow \mathrm{P} \rightarrow \mathrm{S} \rightarrow \mathrm{R})$. If the strategies in this game represent different species or strains, it is immediately obvious how this cyclic competition structure could promote coexistence, because although competition may be intense between pairs of strategies, none is superior or inferior to any other at the community level.

In addition to a rich literature exploring the theoretical consequences of non-transitive competition (Gilpin 1975, May and Leonard 1975, Karlson and Jackson 1981, Tainaka 1988, 2001, Durrett and Levin 1997, 1998, Huisman and Weissing 1999, 2001b, Huisman et al. 2001, Frean and Abraham 2001, Czárán et al. 2002, Laird and Schamp 2006, 2008, 2009, Rojas-Echenique and Allesina 2011), there is a growing number of examples of non-transitive coexistence in a variety of biological systems, including among colour/behavioural morphs in lizards (Sinervo and Lively 1996, Sinervo et al. 2007), benthic invertebrates (Buss and Jackson 1979, Buss 1980, Jackson 1983), algae (Huisman and Weissing 2001a), plants (Lankau and Strauss 2007), and bacteria (Kerr et al. 2002, Kirkup and Riley 2004). The latter example, in which bacteria play RPS, is my focus here.

Kerr et al. (2002) deploy complementary simulation models and experiments to describe non-transitive coexistence of colicinogenic $(C)$, susceptible $(S)$, and resistant $(R)$ strains of Escherichia coli. The $\mathrm{C}$ strain produces colicin, a toxin to which $S$ is sensitive but $R$ is not. In pairwise competition, then, $\mathrm{R}$ outcompetes $\mathrm{C}$ due to a growth-rate advantage, which stems from $\mathrm{R}$ not having to pay the metabolic cost to produce colicin. Similarly, $\mathrm{S}$ outcompetes R because $S$ does not pay the cost of toxicity or resistance. Finally, $\mathrm{C}$ outcompetes $\mathrm{S}$ through the direct action of toxicity. This closes the non-transitive loop $(C \rightarrow R \rightarrow S \rightarrow C)$, leading to the potential for strain coexistence.

Interestingly, however, Kerr et al. (2002) show that this CSR scenario leads to strain coexistence in spatially structured populations (cultured on static agar plates), but not in well-mixed populations (cultured on experimentally 
mixed plates or in a liquid medium). Their lattice-based simulation models also exhibit this strong spatial dependence (at least for certain parameter values; other parameter values lead to monocultures in the models even when space is explicit and the criteria for non-transitive replacement among $\mathrm{C}, \mathrm{S}$ and $\mathrm{R}$ are met). In both the experimental and simulated spatial systems, local clumps of strains form spontaneously and follow each other around their respective competitive arenas in a 'balanced chase' (Kerr et al. 2002). In the well-mixed systems, the omnipresence of $\mathrm{C}$ leads to the rapid extinction of $S$ followed shortly by the extinction of C itself (but also see Müller and Gallas 2010 , who show that the winning strain in well-mixed systems is context dependent).

These patterns raise the question of what facet of spatial competition is critical in allowing the coexistence of nontransitively competing strains of E. coli. Specifically, does spatial structure promote coexistence merely because it reduces the number of neighbours with which individuals interact, allowing members of each strain to effectively 'hide' from their respective enemies? Or, alternatively, do the higher-order properties of spatial networks - in particular, their high average path length ('degrees of separation') and clustering ('cliquishness'; Watts and Strogatz 1998) - also play a role by promoting the spontaneous emergence of local patches of strains?

Szolnoki and Szabó (2004) and Szabó et al. (2004), building on seminal work on 'small-world networks' by Watts and Strogatz (1998), provide preliminary answers to these questions for a simplified RPS game in which the replacement rates between the pairs of strategies are identical. In this situation, spatial lattice models predict stationary strategy concentrations of R, P and $S$ of $1 / 3$ (Szabó et al. 2004). However, when the spatial nature of the interaction structure is relaxed by replacing at least a critical number of local neighbourhood connections with random long-range connections, oscillatory dynamics occur spontaneously (Szabó et al. 2004). The magnitude of these oscillations increases as the population interaction structure gets farther away from a spatial lattice and more like a random regular graph. Thus, in finite populations, extinction leading to monoculture should be more likely when spatial interactions are gradually eliminated while preserving the degree distribution of the interaction network (i.e. the number of other individuals with which an individual interacts). In other words, spatially explicit interactions are important for strategy coexistence in the simplified RPS game, beyond their effects in limiting the number of neighbours with which a given individual interacts.

Here, I apply a similar approach to the CSR model. For lattices with four different degrees (i.e. edges per node), I confirm that spatial structure leads to strain coexistence for certain model parameters. I then show that coexistence is not possible in random regular graphs with the same degree distributions as their lattice counterparts. Finally, I use regular small-world networks with the same degree distributions to show that the transition between coexistence and monoculture occurs when only small deviations from spatial population interaction structure are introduced. These results emphasize the explicit role of space, rather than limited interactions, as being decisive in allowing the coexistence of $\mathrm{C}, \mathrm{S}$ and $\mathrm{R}$ in this model system.

\section{Methods}

I describe a model that elaborates on Kerr et al. (2002), which, in turn, builds on earlier models (Durrett and Levin 1997) and empirical studies (Chao and Levin 1981). The methods of Szolnoki and Szabó (2004) and Szabó et al. (2004) are also critical to the model's development. Kerr et al.'s model matches their empirical results extraordinarily well, suggesting that they do a good job of capturing the essential elements of their system's dynamics. With this in mind, I contend that insight into the original bacterial system can be gleaned by investigating modifications to their model. This guiding philosophy is important here, because I examine situations that are probably counterfactual (e.g. bacteria interacting on what are essentially social networks) in order to better understand why RPS coexistence is possible in real situations (i.e. bacteria interacting with local neighbours on surfaces).

Competitive interactions are represented by graphs (in the graph-theoretical sense; Lieberman et al. 2005). Nodes represent individuals (or, in an alternative interpretation, colonies), with edges connecting nodes whose interaction neighbourhoods overlap. Spatial systems correspond to lattices in which individuals interact only with their $k$ nearest neighbours. I investigate the familiar von Neumann $(k=4)$ and Moore $(k=8)$ lattices as well as those where each individual has $k=3$ or $k=6$ neighbours. Following Kerr et al. (2002), the lattices are square, with a side length of $L=250$ nodes (i.e. a total of $N=L^{2}=62500$ nodes). Further, the lattices have periodic boundaries to avoid edge effects and to ensure that every node has exactly $k$ neighbours.

My general approach to investigate the role of space is to compare the outcome of non-transitive competition on lattices with the outcome of non-transitive competition on networks that retain many traits of lattices (in particular the degree distribution) while gradually relaxing the traits corresponding to spatial structure. To this end, non-spatial networks are also investigated by severing lattice edges and randomly reassembling the resultant half-edges so that the degree distribution of the original lattice is preserved (i.e. every node is still connected to exactly $k$ other nodes), but the higher-order spatial structure associated with lattices is diminished or eliminated (Fig. 1). If all the edges are severed, the resulting network is a random $k$-regular graph (hereafter a 'random regular graph'). If only a proportion, $Q \in[0,1]$, of the edges are severed, the resulting network is also a $k$-regular graph, but with small-world properties (hereafter a 'small-world network'; Watts and Strogatz 1998). Thus, $Q$ represents the degree of 'quenched randomness' in the network (Szabó et al. 2004, Szolnoki et al. 2008). Further, small-world networks represent the continuum joining lattices $(Q=0)$ and random regular graphs $(Q=1)$. (After random regular graphs and small-world networks are generated algorithmically, an additional model step checks that they are connected; i.e. that every node is linked to every other node by a finite chain of intermediary nodes 
(a)

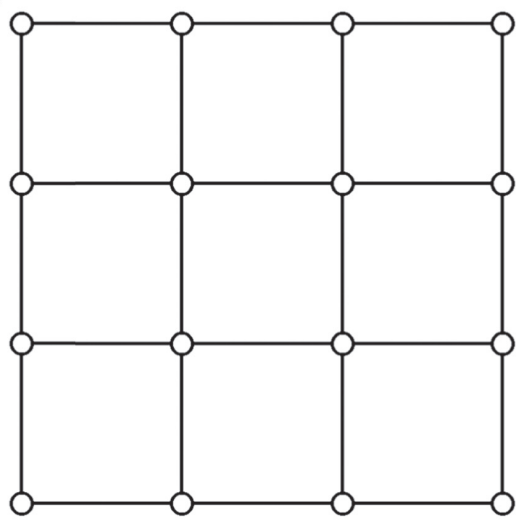

(c)

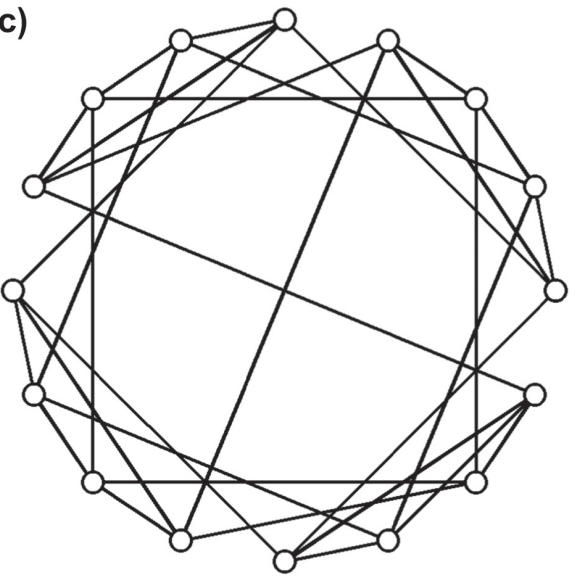

(b)

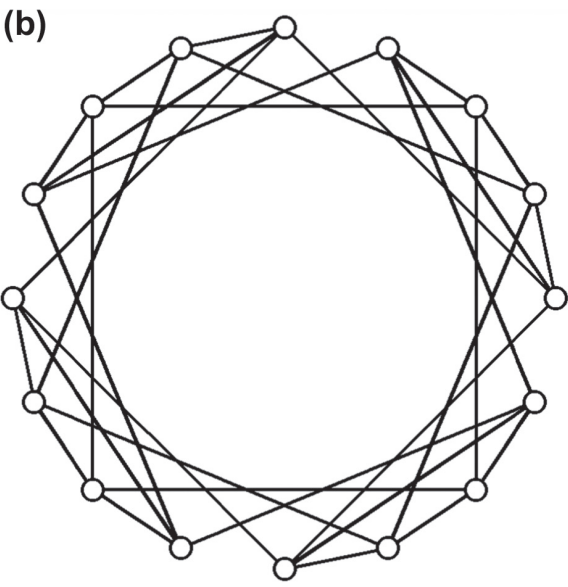

(d)

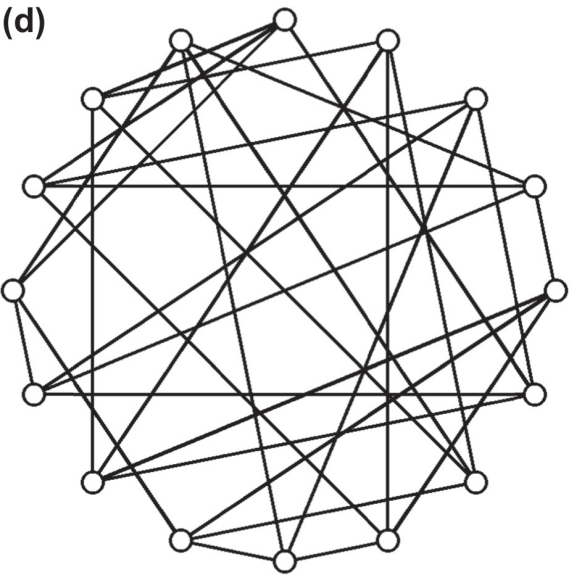

Figure 1. Example graphs representing population interaction structure. (a) A lattice with every individual (node) having exactly $k=4$ neighbours (nodes connected with edges to the focal node). Boundaries are periodic; the top row is connected to the bottom row and the left column is connected to the right column. (b) The same lattice in (a), with nodes rearranged to facilitate comparison with (c) and (d). (c) Small-world network created from (b) by randomly severing $Q=1 / 8$ of its edges and randomly rewiring the resulting half-edges. (d) Random regular graph created from (b) by randomly severing all its edges and randomly rewiring the resulting half-edges. These graphs have $N=4^{2}=16$ nodes; the actual graphs used in the models in this study have $N=250^{2}=62500$ nodes.

and edges. Disconnected graphs are discarded and replaced, although graph connectedness is virtually certain in random graphs of the size investigated here.)

Each node is of one of four different flavours, allocated randomly and independently at the start of each model run: C, S, R and empty nodes. In every time-step, a node is selected at random. If the focal node is empty, it is randomly replaced by a clone of one of its $k$ neighbours, including empties (replacement by a clone of an empty node simply refers to the situation where colonization fails to occur). If the focal node is occupied, the occupant dies with probability $d_{\mathrm{C}}, d_{\mathrm{S}}$, or $d_{\mathrm{R}}$ for C-, S- and R-nodes, respectively, and the node becomes empty (Kerr et al. 2002). The death probabilities $d_{C}$ and $d_{R}$ are constants. However, $d_{\mathrm{S}}=d_{\mathrm{S} 0}+\tau f_{\mathrm{C}}$, where $d_{\mathrm{S} 0}$ is the constant 'baseline' death probability of $S$ in the absence of $C$ neighbours, $f_{\mathrm{C}}$ is the fraction of $\mathrm{C}$ neighbours linked to the focal $S$, and $\tau$ is the intensity of toxicity of these $\mathrm{C}$ neighbours. Meanfield analysis indicates that $d_{\mathrm{S} 0}<d_{\mathrm{R}}<d_{\mathrm{C}}<\left(d_{\mathrm{S} 0}+\tau\right) /$ $(1+\tau)$ to ensure non-transitivity (Kerr et al. 2002). Following Kerr et al. (2002) and others (Károlyi et al.
2005, Müller and Gallas 2010), I consider $d_{\mathrm{C}}=1 / 3$, $d_{S 0}=1 / 4$ for a wide range of combinations of $d_{\mathrm{R}}$ and $\tau$ that satisfy the non-transitivity criterion, and which ensure that all probabilities remain between 0 and 1 .

$N$ time-steps represent a single model generation ('epoch' in Kerr et al. 2002), such that every node is the focal node once per generation, on average. (Naturally the realized number of times that a node is the focal node will follow a Poisson distribution.) The models are run until monoculture or until a 'coexistence criterion' is met. The coexistence criterion is typically $10^{4}$ generations $\left(10^{5}\right.$ generations in the case of small-world networks in which the trajectory towards coexistence or monoculture often takes longer to resolve). There is no mutation (cf. Kerr et al.).

Lattice models are run first and recapitulate the results of Kerr et al. (2002). That is, there are regions of parameter space that allow CSR coexistence in the spatial arena. Simulations on random regular graphs are run next and, by contrast, exhibit no coexistence for any of the parameter values examined. Therefore, for a given set of parameter values that allows for coexistence in the spatial 
setting, there is a transition to monoculture as the degree of quenched randomness, $Q$ (which can be profitably thought of as an inverse measure of 'spaciness') increases from 0 to 1 . This transition is highly relevant to my purposes here: if the critical $Q$ value is small (close to 0 ), then the preservation of spatial structure per se is crucial to strain coexistence; if the critical $Q$ value is large (close to 1 ), then limited interactions - and not spatial structure - are sufficient to promote strain coexistence in this model system. The critical value of $Q, Q_{50}$, is defined as the maximum value of $Q$ where coexistence is expected at least half the time, as determined by logistic regression.

\section{Results}

In lattice models, long-term coexistence is possible for some parameter values but not for others (Fig. 2). Where it is possible, cyclic dynamics are observed as clumps of $\mathrm{C}, \mathrm{S}$ and $\mathrm{R}$ strains follow each other around the arena in a 'balanced chase' (Kerr et al. 2002). In the parameter space investigated, there is a large region where coexistence lasts for at least $10^{4}$ generations (Fig. 3). In the region where coexistence does not occur, the eventual winning monoculture (C, $\mathrm{S}$ or $\mathrm{R})$ depends on the specific parameter values examined (Fig. 3).

In random regular graphs, long-term coexistence does not occur for any of the parameter values examined (Fig. 4). Rather, every population examined (a total of 129200 replicates) exhibits a monoculture of $\mathrm{C}, \mathrm{S}$ or $\mathrm{R}$ within $10^{4}$ generations (Fig. 4).
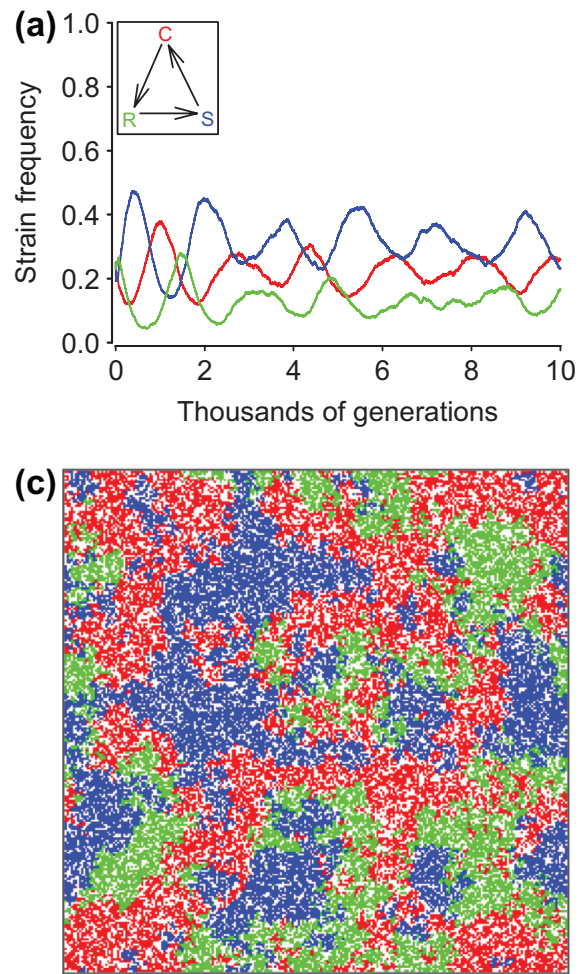

Thus, at least some degree of 'spaciness' is a necessary, albeit not always a sufficient condition to allow for the coexistence of C, S and R in this model. Figure 5 shows the transition between coexistence and monoculture for an example suite of parameters for which coexistence is always observed in the spatial setting (i.e. the same parameters as in Fig. 2a). When the model is run on former lattices with proportion $Q_{50}$ of the edges rewired, large oscillations ensure that monoculture and coexistence are equally likely, at least for the given coexistence criterion (e.g. Fig. 6). These oscillations are much larger than those found on the lattice (compare Fig. 6 with Fig. 2a).

When $Q_{50}$ values are calculated for parameter combinations for which lattices allow for the potential for strain coexistence, it is clear that relatively small departures from the spatial setting destroy this potential. For the parameter values and degree distributions examined, the odds of strain coexistence are already worse than 50-50 when less than $1 \%$ of the edges in a lattice are randomly severed and reassembled (Fig. 7). (This is true whether the coexistence criterion is $10^{4}$ or $10^{5}$ generations, although only the latter is shown in Fig. 7).

\section{Discussion}

The role of space in promoting species or strain coexistence is a general problem in community ecology, and more specifically in the case of non-transitive competition (Durrett and Levin 1997, 1998, Szabó and Czárán 2001, Czárán et al. 2002, Kerr et al. 2002, Reichenbach et al.
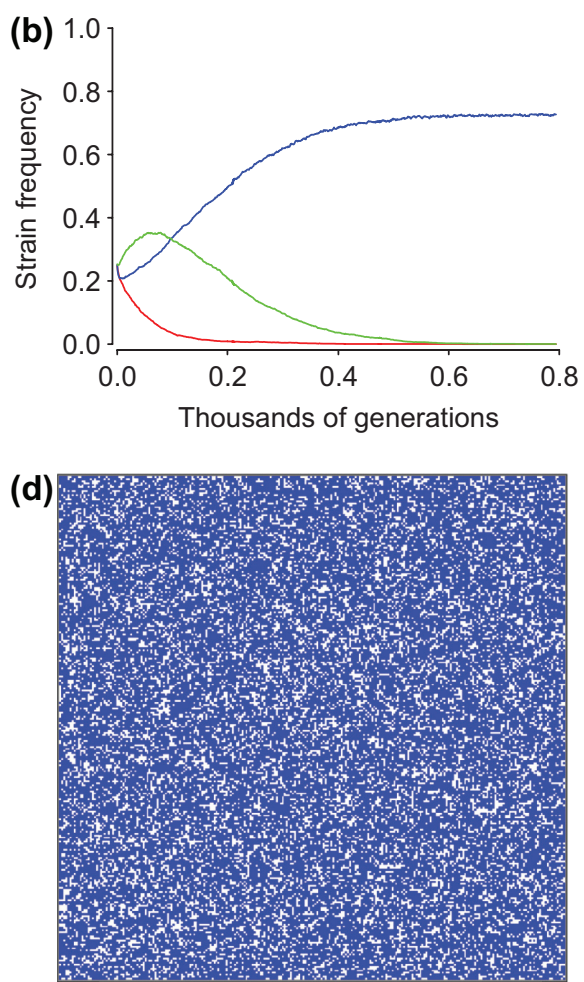

Figure 2. (a, b) Example time series and (c, d) corresponding lattices $(N=62500, k=4)$ after 10000 generations for two sets of parameter values. In both the time series and the lattices, red $=\mathrm{C}$, blue $=\mathrm{S}$, and green $=\mathrm{R}$; in the lattices, white $=$ empty node. $(\mathrm{a}, \mathrm{c})$ $d_{\mathrm{C}}=1 / 3, d_{\mathrm{S} 0}=1 / 4, d_{\mathrm{R}}=3 / 10, \tau=3 / 5$. (b, d) $d_{\mathrm{C}}=1 / 3, d_{\mathrm{S} 0}=1 / 4, d_{\mathrm{R}}=11 / 40, \tau=2 / 5$. 

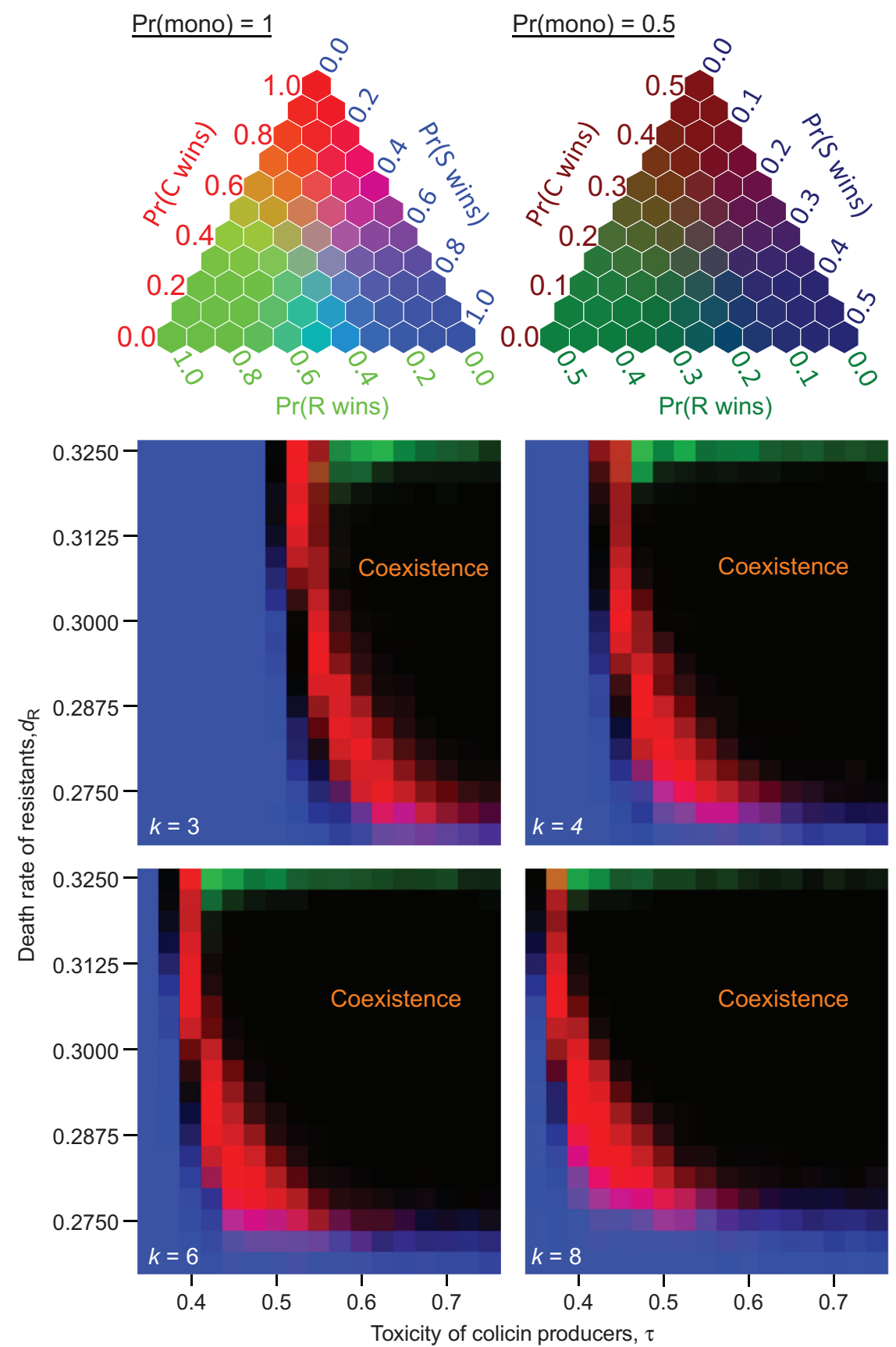

Figure 3. Probability of strain coexistence and monoculture (for $d_{\mathrm{C}}=1 / 3, d_{S 0}=1 / 4$, and a range of values of $d_{\mathrm{R}}$ and $\tau$ ) for competition on lattices with $N=62500$ nodes and $k=3, k=4, k=6$, or $k=8$ neighbours per node. As shown in the legends, the colour at each pair of parameter coordinates specifies the probabilities of C, S and R monocultures within $10^{4}$ model generations (the coexistence criterion) over 100 model runs, with red representing a $\mathrm{C}$ monoculture, blue representing an $\mathrm{S}$ monoculture, and green representing an $\mathrm{R}$ monoculture. The intensity of the colour represents the probability that a monoculture occurs at all (' $\left.\operatorname{Pr}(\mathrm{mono})^{\prime}\right)$; thus, two example legends are given, one for $\operatorname{Pr}(\operatorname{mono})=1$ and one for $\operatorname{Pr}($ mono $)=0.5$. The large, labeled, black regions represent parameter space in which three-strain coexistence occurs. The narrow, dark regions between monoculture regions (e.g. on the C-S border) represent regions where one strain is already extinct but the identity of the inevitable monoculture strain is not resolved within $10^{4}$ generations.

2007, Vellend and Litrico 2008, Laird and Schamp 2008, 2009, Rojas-Echenique and Allesina 2011). One important question that emerges from this work is whether any putative effects of space can be explained simply by the limited interactions experienced by spatially interacting agents, or, alternatively, whether space per se (i.e. interaction specifically with nearest neighbours) is critical. Work on a simplified model of rock-paper-scissors competition suggests it is the latter (Szolnoki and Szabó 2004, Szabó et al. 2004, also see Zhang et al. 2009).

Here, I apply and extend these findings to Kerr et al.'s (2002) model of non-transitive competition in colicinogenic, susceptible, and resistant $E$. coli strains - a model that very closely matches empirical results in this system. 

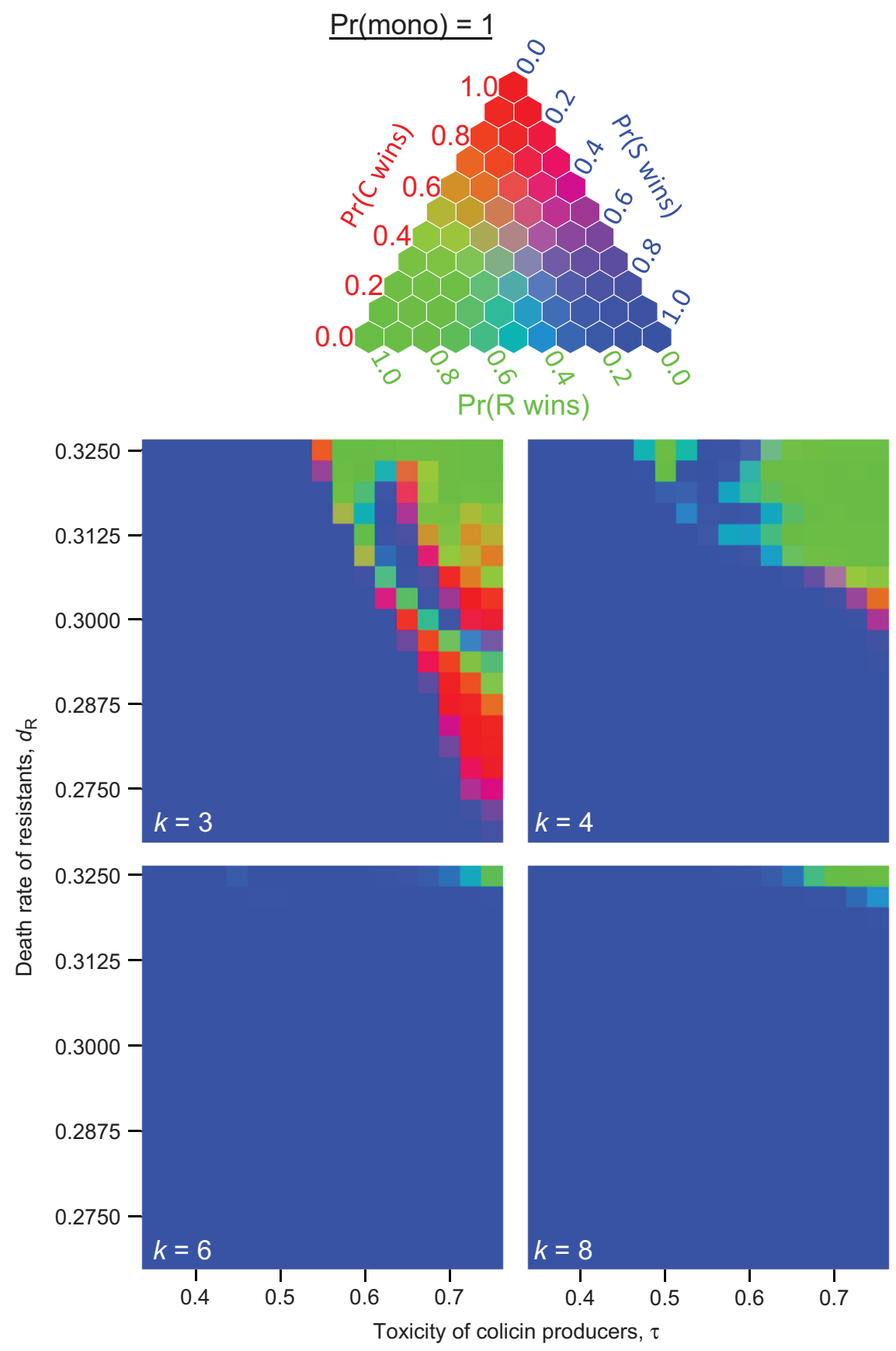

Figure 4. Probability of strain coexistence and monoculture (for $d_{\mathrm{C}}=1 / 3, d_{\mathrm{S} 0}=1 / 4$, and a range of values of $d_{\mathrm{R}}$ and $\tau$ ) for competition on random regular graphs with $N=62500$ nodes and $k=3, k=4, k=6$, or $k=8$ neighbours per node. As shown in the legend, the colour at each pair of parameter coordinates specifies the probabilities of $\mathrm{C}, \mathrm{S}$ and $\mathrm{R}$ monocultures within $10^{4}$ model generations (the coexistence criterion) over 100 model runs, with red representing a $\mathrm{C}$ monoculture, blue representing an $\mathrm{S}$ monoculture, and green representing an $\mathrm{R}$ monoculture. The intensity of the colour represents the probability that a monoculture occurs at all (' $\operatorname{Pr}(\mathrm{mono})$ '); in contrast to lattices (Fig. 3), on random regular graphs, monocultures always occur at all parameter combinations examined (i.e. no coexistence). Thus, only a single legend is provided (for $\operatorname{Pr}(\operatorname{mono})=1)$.

I show that while strain coexistence is often possible in (spatial) lattice models, coexistence disappears when competition takes place on random regular graphs and even in small-world networks with any more than a very small degree of quenched randomness. In the spatial setting, local interactions and dispersal lead to the spontaneous emergence of a 'fluid mosaic of patches' that undergo a 'balanced chase' (Kerr et al. 2002); breaking down the spatial structure, even by a relatively small amount, leads to ever-larger oscillations in the $\mathrm{C} \rightarrow \mathrm{R} \rightarrow \mathrm{S} \rightarrow \mathrm{C}$ dynamics, eventually leading to a monoculture of one strain or another (with the victor depending on the relative competitive abilities of the C, S and R strains). Thus, these results support Kerr et al.'s assertion of the importance of space, and emphasize that the topology of interaction networks - not just their degree distribution - is important in determining 


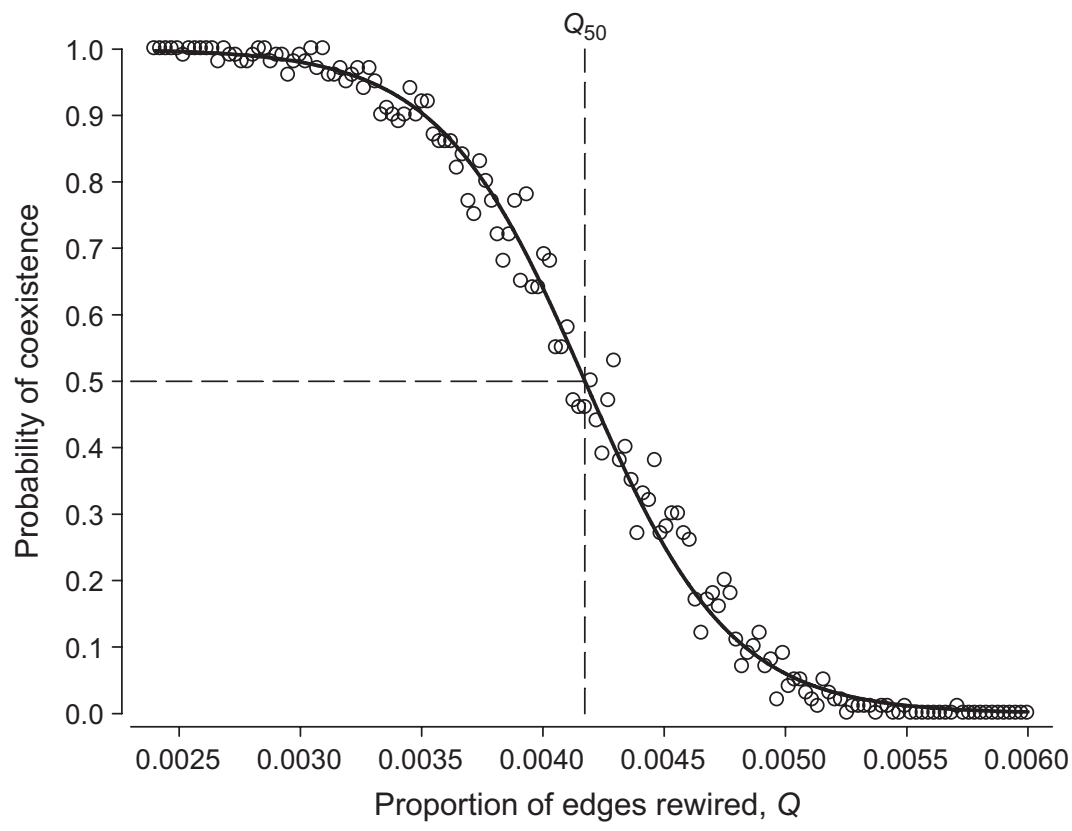

Figure 5. An example of the relationship between the probability of strain coexistence and the degree of quenched randomness, $Q$. The coexistence criterion is a minimum of $10^{5}$ model generations. Each point is the result of 100 model runs. The equation for the line, found using logistic regression, is given by $p=\frac{\exp (13.8847-3327.1 Q)}{1+\exp (13.8847-3327.1 Q)}$. $Q_{50}$ is the value of $Q$ where the predicted probability of coexistence is 0.5 (here, $Q_{50}=0.00417$ ). Parameter values: $N=62500, k=4, d_{\mathrm{C}}=1 / 3, d_{\mathrm{S} 0}=1 / 4, d_{\mathrm{R}}=3 / 10$, $\tau=3 / 5$; note that these are the same as in Fig. 2a.

coexistence. More generally, these findings complement the broader class of models that show how relaxing spatial network structure (e.g. by increasing mobility or mixing; Károlyi et al. 2005, Reichenbach et al. 2007) eventually leads to a collapse of diversity in many RPS systems. Further, the fact that only minor relaxation is needed to effect such a collapse suggests that spatially mediated RPS coexistence is likely to be most important when relatively immobile competitors are confined to a stable substrate.

While lattice models and global- or local-mixing models have close analogues in real bacterial systems (e.g. bacteria cultured in static or well-mixed growth medium, respectively; Kerr et al. 2002), random regular graphs and small-world networks probably do not. (Bacteria do not join Facebook.) Nevertheless, I argue that models involving these unrealistic interaction structures are still relevant to understanding real bacteria because they allow a vantage from which we can contrast interaction structures whose link to real bacteria is already established (e.g. Fig. 3 vs Fig. 4 and Fig. 7). Indeed, this is an interesting example of a situation where a model can illuminate aspects of a biological system that are difficult to understand by directly manipulating the system itself.
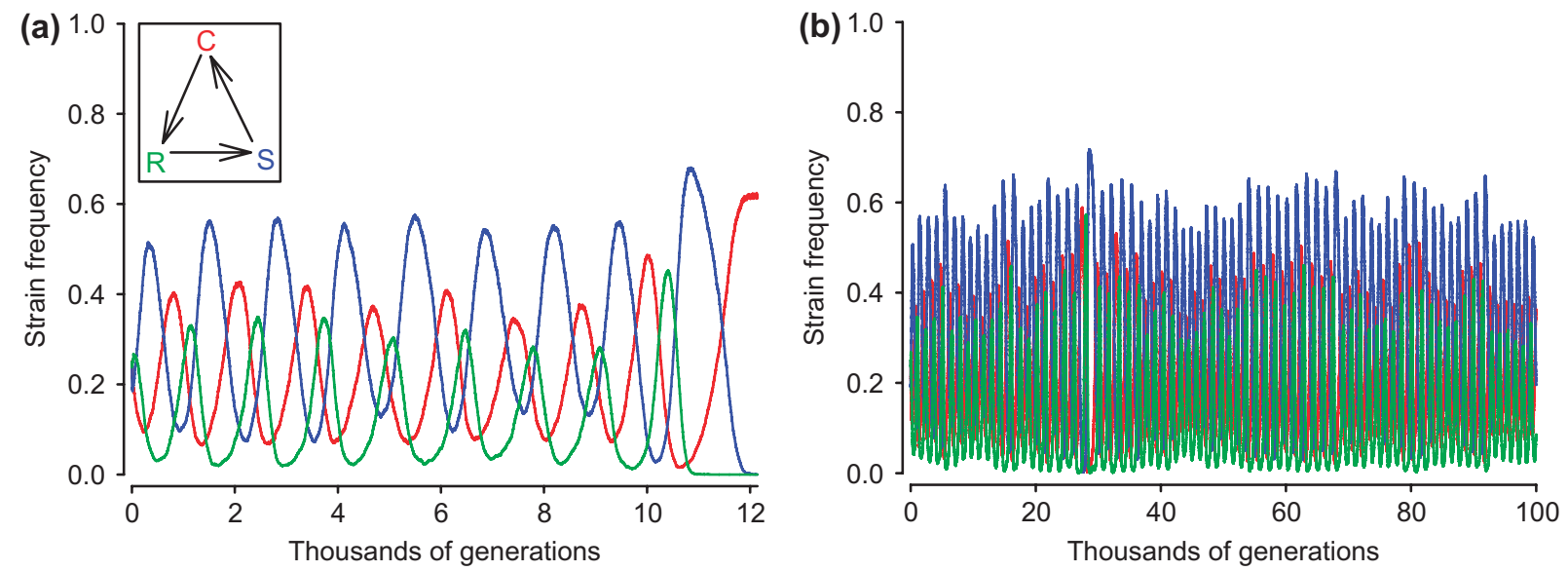

Figure 6. Two example time series when $Q=Q_{50}=0.00417$. (The two runs use different small-world networks with the same degree of quenched randomness.) Parameter values: $N=62500, k=4, d_{\mathrm{C}}=1 / 3, d_{\mathrm{S}_{0}}=1 / 4, d_{\mathrm{R}}=3 / 10, \tau=3 / 5$; see Fig. 5. (a) Monoculture of C occurs within 12141 generations. (b) Coexistence occurs for more than $10^{5}$ generations. 


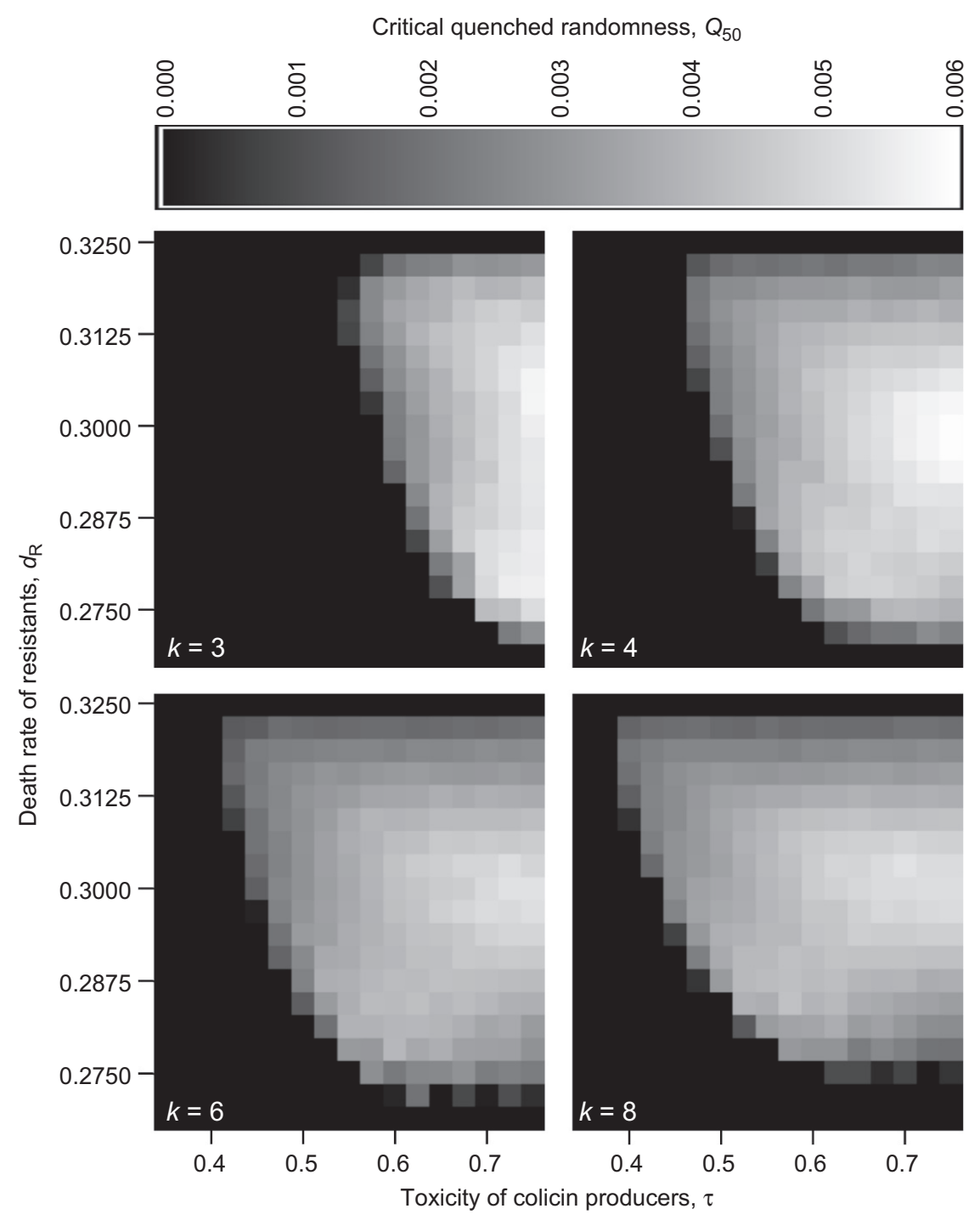

Figure 7. Values of $Q_{50}$ (for $d_{\mathrm{C}}=1 / 3, d_{\mathrm{S} 0}=1 / 4$, and a range of values of $d_{\mathrm{R}}$ and $\tau$ ) for competition on small-world networks with $N=62500$ nodes and $k=3, k=4, k=6$, or $k=8$ neighbours per node. Lighter shades represent a greater $Q_{50}$. Black regions represent parameter combinations where coexistence is not possible even in the spatial setting (Fig. 3); in this case there is no value of $Q$ for which coexistence is expected $50 \%$ of the time.

Of course, many other systems, particularly those involving human interactions or technological artifacts, but also in non-human biological entities such as pathogen-transmission networks and the neural networks of C. elegans, do have small-world properties (Watts and Strogatz 1998). In light of this, an obvious extension of the results discussed here would be to test the prediction that such networks are increasingly unlikely to admit the coexistence of replicators (e.g. behavioural strategies, ideas, pathogens, etc.) playing variations of the rock-paperscissors game as the degree of quenched randomness increases (Szolnoki and Szabó 2004, Szabó et al. 2004).

Acknowledgements - I gratefully acknowledge Paul Leventis, Lee Chang, Soroosh Yazdani and Eitan Bonderover for technical assistance. Brandon Schamp and subject editor Stefano Allesina provided helpful comments on the manuscript. Funding was provided by the Natural Sciences and Engineering Research Council of Canada and by the University of Lethbridge.

\section{References}

Buss, L. W. 1980. Competitive intransitivity and size-frequency distributions of interacting populations. - Proc. Natl Acad. Sci. USA 77: 5355-5359.

Buss, L. W. and Jackson, J. B. C. 1979. Competitive networks: nontransitive competitive relationships in cryptic coral reef environments. - Am. Nat. 113: 223-234.

Chao, L. and Levin, B. R. 1981. Structured habitats and the evolution of anticompetitor toxins in bacteria. - Proc. Natl Acad. Sci. USA 78: 6324-6328.

Chesson, P. 2000. Mechanisms of species diversity. - Annu. Rev. Ecol. Syst. 31: 343-366.

Czárán, T. et al. 2002. Chemical warefare between microbes promotes biodiveristy. - Proc. Natl Acad. Sci. USA 99: 786-790.

Durrett, R. and Levin, S. 1997. Allelopathy in spatially distributed populations. - J. Theor. Biol. 185: 165-171.

Durrett, R. and Levin, S. 1998. Spatial aspects of interspecific competition. - Theor. Popul. Biol. 53: 30-43.

Frean, M. and Abraham, E. R. 2001. Rock-scissors-paper and the survival of the weakest. - Proc. R. Soc. B 268: 1323-1327. 
Gilpin, M. E. 1975. Limit cycles in competition communities. - Am. Nat. 109: 51-60.

Huisman, J. and Weissing, F. J. 1999. Biodiversity of plankton by species oscillations and chaos. - Nature 402: 407-410.

Huisman, J. and Weissing, F. J. 2001a. Biological conditions for oscillations and chaos generated by multispecies competition. - Ecology 82: 2682-2695.

Huisman, J. and Weissing, F. J. 2001b. Fundamental unpredictability in multispecies competition. - Am. Nat. 157: 488-494.

Huisman, J. et al. 2001. Towards a solution of the plankton paradox: the importance of physiology and life history. - Ecol. Lett. 4: 408-411.

Jackson, J. B. C. 1983. Biological determinants of present and past sessile animal distributions. - In: Tevesz, M. J. S. and McCall, P. L. (eds), Biotic interactions in recent and fossil benthic communities. Plenum Press, pp. 39-76.

Karlson, R. H. and Jackson, J. B. C. 1981. Competitive networks and community structure - a simulation study. - Ecology 62: 670-678.

Károlyi, G. et al. 2005. Rock-scissors-paper game in a chaotic flow: the effect of dispersion on the cyclic competition of microorganisms. - J. Theor. Biol. 236: 12-20.

Kerr, B. et al. 2002. Local dispersal promotes biodiversity in a reallife game of rock-paper-scissors. - Nature 418: 171-174.

Kirkup, B. C. and Riley, M. A. 2004. Antibiotic-mediated antagonism leads to a game of rock-paper-scissors in vivo. - Nature 428: 412-414.

Laird, R. A. and Schamp, B. S. 2006. Competitive intransitivity promotes species coexistence. - Am. Nat. 168: 182-193.

Laird, R. A. and Schamp, B. S. 2008. Does local competition increase the coexistence of species in intransitive networks? - Ecology 89: 237-247.

Laird, R. A. and Schamp, B. S. 2009. Species coexistence, intransitivity, and topological variation in competitive tournaments. - J. Theor. Biol. 256: 90-95.

Lankau, R. A. and Strauss, S. Y. 2007. Mutual feedbacks maintain both genetic and species diversity in a plant community. - Science 317: 1561-1563.

Lieberman, E. et al. 2005. Evolutionary dynamics on graphs. - Nature 433: 312-316.

May, R. M. and Leonard, W. J. 1975. Nonlinear aspects of competition between three species. - SIAM J. Appl. Math. 29: 243-253.
Müller, A. P. O. and Gallas, J. A. C. 2010. How community size affects survival chances in cyclic competition games that microorganisms play. - Phys. Rev. E 82: 052901.

Reichenbach, T. et al. 2007. Mobility promotes and jeopardizes biodiversity in rock-paper-scissors games. - Nature 448: 1046-1049.

Rojas-Echenique, J. and Allesina, S. 2011. Interaction rules affect species coexistence in intransitive networks. - Ecology 92: 1174-1180.

Sinervo, B. and Lively, C. M. 1996. The rock-paper-scissors game and the evolution of alternative male strategies. - Nature 380: 240-243.

Sinervo, B. et al. 2007. Models of density-dependent genic selection and a new rock-paper-scissors social system. - Am. Nat. 170: 663-680.

Szabó, G. and Czárán, T. 2001. Defensive alliances in spatial models of cyclical population interactions. - Phys. Rev. E. 64: 042902 .

Szabó, G. et al. 2004. Rock-scissors-paper game on regular smallworld networks. - J. Phys. A: Math. Gen. 37: 2599-2609.

Szolnoki, A. and Szabó, G. 2004. Phase transitions for rock-scissors-paper game on different networks. - Phys. Rev. E 70: 037102 .

Szolnoki, A. et al. 2008. Diversity of reproduction rate supports cooperation in the prisoner's dilemma game on complex networks. - Eur. Phys. J. B 61: 505-509.

Tainaka, K.-I. 1988. Lattice model for the Lotka-Volterra system. - J. Phys. Soc. Jpn 57: 2588-2590.

Tainaka, K.-I. 2001. Physics and ecology of rock-paperscissors game. - Lect. Notes Comp. Sci. 2063: 384-395.

Tokeshi, M. 1999. Species coexistence: ecological and evolutionary perspectives. - Blackwell.

Vellend, M. and Litrico, I. 2008. Sex and space destabilize intransitive competition within and between species. - Proc. R. Soc. B 275: 1857-1864.

Watts, D. J. and Strogatz, S. H. 1998. Collective dynamics of 'small-world' networks. - Nature 393: 440-442.

Wilson, J. B. 1990. Mechanisms of species coexistence: twelve explanations for Hutchinson's 'Paradox of the Plankton': evidence from New Zealand plant communities. - N. Zealand J. Ecol. 13: 17-42.

Zhang, G.-Y. et al. 2009. Four-state rock-paper-scissors games in constrained Newman-Watts networks. - Phys. Rev. E 79: 062901. 